\title{
Performances in extreme environments: effects of hyper/hypobarism and hypogravity on skeletal muscle
}

\author{
Gerardo Bosco, Vittore Verratti, Giorgio Fanò \\ Department of Basic and Applied Medical Sciences (BAMS), Interuniversity Institute \\ of Myology (IIM), “G. d’Annunzio” University, Chieti-Pescara, Italy
}

\begin{abstract}
Many environmental factors may affect muscle plasticity but some have exclusive characteristics that allow them to play a key role to maintain the muscle capacity to generate force; these factors are: i) the oxygen availability and ii) the load applied to muscle fibres. Hyperbarism is a condition that occurs when a man is subjected to pressure increases. To keep the lungs from collapsing, the air is supplied to him under high pressure which exposes the blood in the lungs to high alveolar gas pressures. Under this condition, the $\mathrm{PO}_{2}$ become sufficiently increased, serious disorders may occur, such as modification of oxygen delivery and/or oxygen availability to permit regular muscle contraction. Also altitude hypobaric hypoxia induces modification of muscle capacity to generate work. Prolonged exposure to high altitude leads significant loss in body mass, thigh muscle mass, muscle fiber area and volume density of muscle mitochondria. Spaceflight results in a number of adaptations to skeletal muscle, including atrophy and early muscle fatigue. Muscle atrophy is observed in a wide range of muscles, with the most extensive loss occurring in the legs, because astronauts are no longer needed to support the body's weight. This review will describe the background on these topics suggesting the strategies to correct the specific muscle changes in presence of environmental stresses, such as the alteration in oxygen-derived signaling pathways or the metabolic consequence of microgravity that may indicate rational interventions to maintain muscle mass and function.
\end{abstract}

Key Words: Skeletal muscle, adaptations, hyperbaric, hypobaric environment, microgravity

European Journal Translational Myology - Myology Reviews 2010; 1 (3): 83-90

\section{Hyperbaric environment}

2. Hypobaric environment

3. Microgravity

Skeletal muscle is a highly plastic tissue that responds to changes in its pattern of activity as consequence of the mechanical and environmental stresses placed upon it. Although the signaling pathways involved in these multiple adaptations are well described, the factors and mechanisms responsible of such processes remain poorly understood [21]. Among the many environmental factors that may affect muscle plasticity, there are some having exclusive characteristics that together with the presence of motor innervation, allows them to play a key role to maintain the muscle capacity to generate force; these factors are: i) the oxygen availability and ii) the load applied to muscle fibres. Because of its high energy demand, skeletal muscle is sensitive to changes in the oxygen availability. ATP is necessary for skeletal muscle function, which is characterized by voluntary maximal or repeated force-generating capacity strictly related to both the amount and type of contractile proteins and the efficiency of the cellular mechanism of excitationcontraction coupling. However, the oxygen-depending processes, are not only correlated to the more efficiently ATP synthesis, but also to production of potentially dangerous derivatives such as reactive oxygen species (ROS). In addition, there is no doubt that microgravity derived from medium and long-term space flight has serious damaging impact upon human physiological systems. These include an extremely dangerous status as the lower limb anti-gravity muscle atrophy, which can be also evident each time that the load applied on the muscle (absence of gravity, tenotomy, bed rest, etc.) is no longer present. This review provides novel information and suggests the strategies aimed at correcting the specific changes induced in muscle, such as the alteration in oxygenderived signaling pathways or the metabolic consequence of microgravity. 
Muscle adaptations in extreme environments

European Journal Translational Myology - Myology Reviews 1 (3): 83-90, 2010.

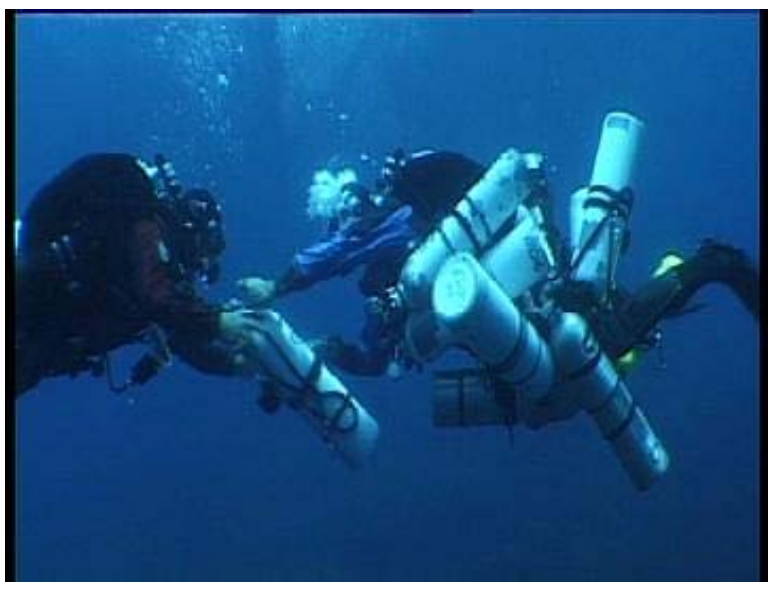

Fig. 1 Deep dive breathing mixture of gases. Courtesy Vittorio Bianchini.

\section{Hyperbaric environment}

When a man is compressed in a closed space or descends beneath the surface of the sea, he is subjected to pressure increases. To keep the lungs from collapsing, the air is supplied to him under high pressure which exposes the blood in the lungs to high alveolar gas pressures. This is known as hyperbarism. Caisson workers for example must work in pressurized areas; all divers enjoy the beneath endure increasing pressure as breath-hold and scuba (self contained underwater apparatus) divers (Fig. 1); or they work at pressure as commercial or military divers; others receive hyperbaric oxygen therapy (HBO) for the treatment of several diseases. High hydrostatic pressure can produce considerable biological effects. According to Fenn [9] enzymes, viruses, and toxins may become inactivated above 1000 atmospheres absolute pressure (ata). Pressure can affect the contractile mechanism of muscle [14], and at very high pressures muscle become rigid and stiff. The effects of pressure become much more relevant for the diver's concern when they interact with the gases in the breathing mixture. As pressure increases, the partial pressures of the gases in the breathing $\mathrm{mix}$ also increase proportionately according to Dalton's law. The amount of each gas dissolved in the blood and tissues depends on the partial pressure. When it becomes sufficiently increased, serious disorders may occur, such as oxygen toxicity, nitrogen narcosis, high pressure nervous syndrome (HPNS) and $\mathrm{CO}_{2}$ poisoning [6]. Few but amazing physiological studies in humans have been performed during these years in superb hyperbaric facilities. They differentiate between the HPNS as manifested during compression and hydrostatic pressure alone. The compression syndrome as part of HPNS is characterized by changes in the EEG, the appearance of both intention and postural tremor, and neuromuscular incoordination, for example, a loss of balance. The HPNS that occurs generally at depths below 31.3 ata is consistent with findings that neuromuscular motor performance is most affected by

\section{Effect of $\mathrm{HBO}$ on interstitial lactate level in muscle during ischemia-reperfusion}

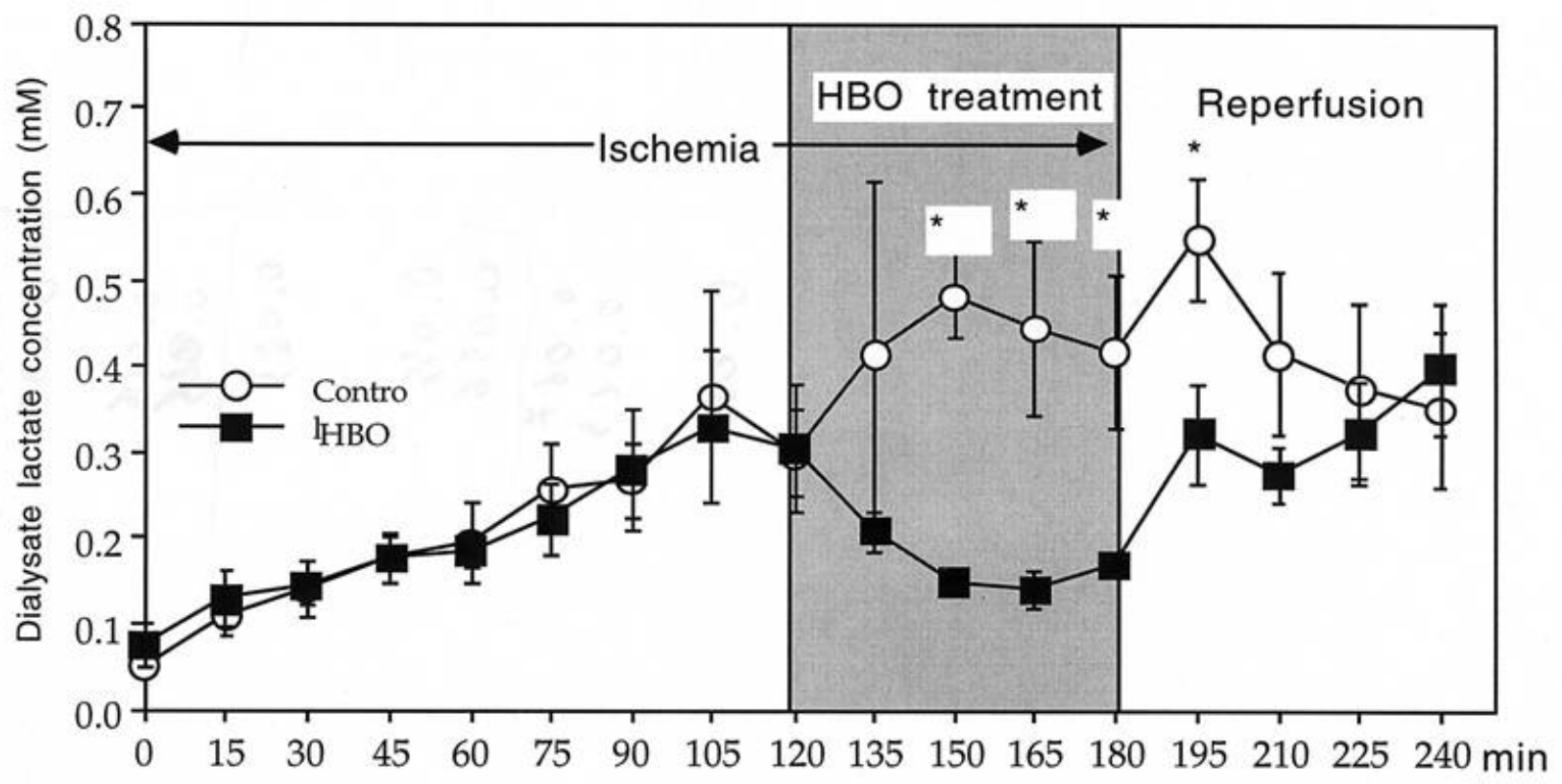

Fig. 2 Effect of HBO on interstitial lactate level in muscle during ischemia reperfusion. From Bosco et al. [5] 
deep dives (breathing a helium-oxygen mixture) while intellectual or cognitive performance is much less affected. The opposite appears to be true in hyperbaric air where intellectual cognitive functions appear to be more degraded than pscymotor skills [28]. How much of this is a function of pressure, and how much is function of breathing helium versus breathing hyperbaric air with its aspects of nitrogen narcosis, is not entirely clear.

There are also some depth-independent effects of the immersion per se as anti-gravity vascular and respiratory pressure effects but these aspects are well detailed in reviews [6,31]. There is a direct effect on muscle contraction as well. In 1981 investigators reported the effect of hyperbaric pressure on isometric muscle contraction and work in intact animals and found the muscle tension increases as the pressure increase up to 35 ata. The rate of development of tension and total work were also increased [26]. The effects of high pressure on the mechanical response of skeletal muscle have been verified [15]. Pressure greatly increases the tension of a single isometric twitch that may reach, under high pressure conditions, the level of a tetanic response. The duration of the twitch is elongated due to an enhanced rate of rise and slowed relaxation. Ranatunga et al. [32] revealed that passive isometric tension in the relaxed state of the muscle is unaffected by increased pressure. In contrast, in the rigor state when the cross-bridges are stable, increased pressure causes an increase of isometric tension. In humans, a lack of torque reduction with higher frequency tetanic stimulation suggested that 6 ata does not impair myofilament kinetics, whereas twitch potentiation may include changes in excitationcontraction coupling [3]. Two studies of human muscle biopsy are reported in breath-hold divers. They demonstrate that prolonged habitual cold-water immersion may induce a decrease in fibre size and an increase in capillarity in female human skeletal muscle but this alteration has been decreased by prolonged habitual breath-hold diving [2,30]. Pressure has similar effects on skeletal and cardiac muscles. Both muscles exhibit a considerable positive inotropic pressure effect at steady state, most probably due to the impedance of various mechanism of $\mathrm{Ca} 2+$ removal from the cytosol either into cellular or extracellular compartments [12]: Increased atmospheric pressure alone is detrimental to physical performance, but this is counteracted by the elevation of inspired oxygen pressure. In most studies there were consistent reports of increased work tolerance with hyperoxia $[11,24]$. It has usually been assumed that the reduction of lactate level during hyperoxia is a result of alleviation of the anaerobic conditions in working muscles (Fig. 4). Another theory is that oxygen may exert its effect directly on the enzymes of the glycolytic pathways. It has been demonstrated the ammonia levels post exercise under

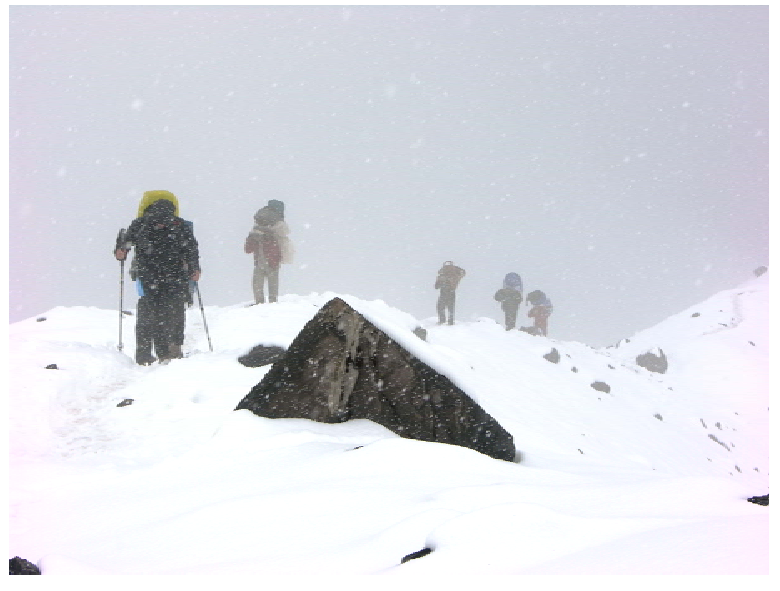

Fig. 3 Himalaya Manaslu Project. Interuniversitary Institute of Myology. Coutesy from expedition team.

HBO were much lower than the corresponding values during normobaric oxygen. Lactate levels after exercise were lower during exercise under oxygen and more pronounced with HBO. There was a fall of glucose with exercise under $\mathrm{HBO}$, suggesting inhibition of glycolysis, which is a contributory factor to the rise in the level of ammonia. These studies confirm the findings of a fall of lactate and pyruvate during exercise under HBO [4]. Recently it has been demonstrated how HBO (2.8 ata) decreases lactate levels in the biceps femoris muscle of rats (. Using a microdyalis technique, authors have been able to monitor lactate as reported in Fig. 2, glucose and glycerol in real time during ischemia-reperfusion injury [35].

In the Atlantis dive series (Fig. 2) arterial blood gases were obtained at rest and during exercise at $650 \mathrm{~m}$ (66 ata in hyperbaric chamber) in humans breathing trimix $\left(\mathrm{He}-\mathrm{N}_{2}-\mathrm{O}_{2}\right)$. The investigators showed hypercapnia, respiratory acidosis, and a new finding was that at depth, arterial lactate levels were higher at rest and during exercise [5]. Recently it seems to be very intriguing the role of respiratory muscle training to avoid the work load of breathing at depth [33]. In conclusion only HBO reduces fatigue and increases physical endurance, mainly due to its effect in reducing the increase of lactic acid production and associated with physical therapy might have several advantages.

\section{2- Hypobaric environment}

Paul Bert in his most famous book "La Pression Barométrique" summarized his work on the effects of barometric pressure upon life. The fundamental physiological effect of decreasing barometric pressure is due to the concomitant fall of the $\mathrm{O}_{2}$ partial pressure. The effects of lowering or raising the barometric pressure can be countered by increasing or decreasing the $\mathrm{O}_{2}$ fraction in the air. Therefore, it is clear that the 
Body mass

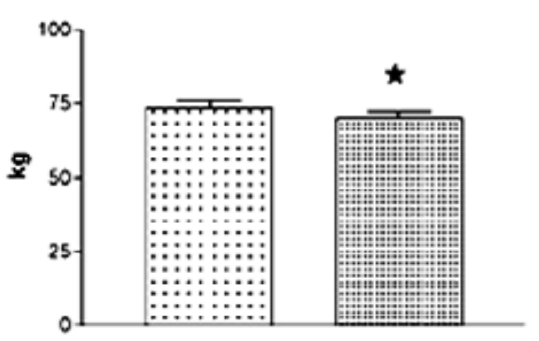

Fiber area

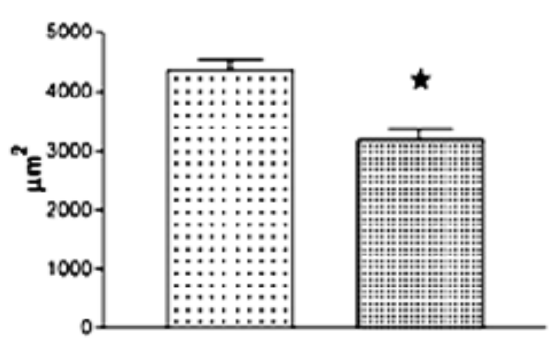

Thigh muscle mass

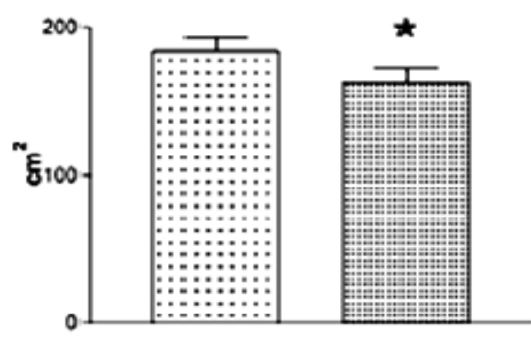

Mitochondria

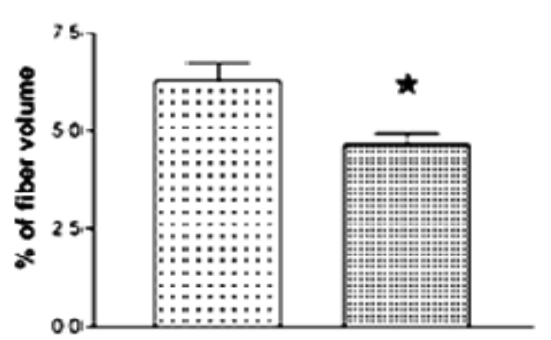

$\mathrm{VO}_{2 \max }$

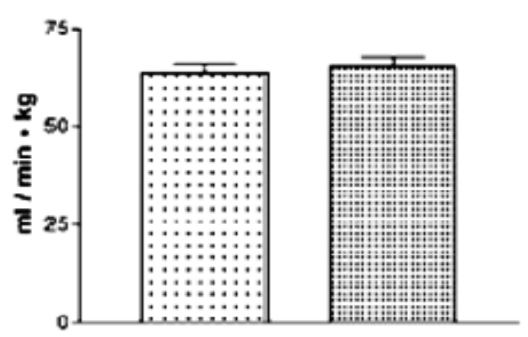

$A_{W}(f, c)$

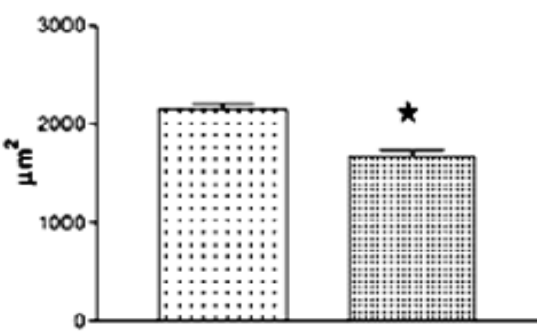

Fig. 4. Changes in body mass, thigh muscle mass determined by computer tomography, $\mathrm{O}_{2}$ max, muscle fibre area, volume density of mitochondria, muscle fibre area perfused by one capillary [AN $(f, c)]$, in members of an expedition to Mt. Everest. Bright columns, before departure to the Himalayas; dark columns, after returning to Switzerland from Margaria et al [24].

main factor related to the barometric pressure change remains the hypoxia, since oxygen inhalation or recompression lead to a quick recovery [7]. Many studies analyzed the effects of short and medium time exposure to hypobaric conditions in people living at sea level while only little is known about functional adaptations to chronic hypobaric exposure of no conditioned population [8].

High altitude living produces physiological changes, including skeletal muscle capacities, as consequence of adaptation to chronic hypobaric-hypoxemic conditions (Fig. 3). Although much is known about these physiologic changes, no evident separation has been made regarding the difference between genetic and acquired adaptations.

As described twenty years ago from Cerretelli's group $[17,18]$, a consequence of enhanced catabolism the prolonged exposure to hypoxia led to a statistically significant loss in body mass, thigh muscle mass, muscle fibre area and volume density of muscle mitochondria (Fig. 2) . The decrease in cross-sectional muscle fibre area was combined with an increase in capillary density, leading to a significant reduction of muscle fibre volume supplied by one capillary. The decrement in mitochondrial volume density was confirmed by statistically significant decreases of enzyme activities responsible for the aerobic-oxidative metabolism [10]. Since this decrease was combined with an increase of enzyme activities involved in anaerobic glycolysis, Howald and Hoppeler [16] have concluded that prolonged exposure to hypoxia together with strenuous exercise at high altitude induces an aerobic to anaerobic shift of muscle energy metabolism. Altitude hypobaric hypoxia induces modification of muscle protein synthesis and pattern as well. Repetitive muscle work in moderate quote with concomitant recovery in a well-oxygenated atmosphere elevates the structural components of local aerobic capacity by increased mitochondrial protein expression and modifications toward reduced regulation of ion gradients. Conversely, permanent hypoxia of muscle causes a net loss of mitochondria, which is compensated for by improved capillary perfusion due to a loss of contractile material. The lowering of intramuscular oxygen tension in combination with non identified contraction-related signals is the driving force for elevated angiogenic and mitochondrial protein expression with endurance training [13]. Hypobaric altitude hypoxia training can improve endurance performance. Several modalities of altitudehypoxic training have been developed to provide the best compromise between hypoxic acclimatization and maintaining high intensity training in the face of a reduced $\mathrm{VO}_{2} \max$ [36]. Prolonged exposure to high altitude leads to reduced muscle mass and performance. The fall in muscle mass follows a reduction in fiber size. Hypoxia alone does not alter capillary number and geometry in skeletal muscles of 
Muscle adaptations in extreme environments

European Journal Translational Myology - Myology Reviews 1 (3): 83-90, 2010.

mammals at altitude. Alterations in fiber size and aerobic enzymes depend on a number of additional factors, including activity and the level of hypoxia. With training at altitude, fiber capillary number and aerobic enzymes are increased, indicating that muscle potential for plasticity is conserved in hypoxia. Capillary number and geometry are altered in muscles of several species of birds native or exposed to higher altitude. Therefore, the capillary growth can occur in skeletal muscle in response to chronic exposure to high altitude [25]. Sustained exposure to severe hypoxia has detrimental effects on muscle structure. Short-term effects on skeletal muscle structure can readily be observed after 2 months of acute exposure of lowlanders to severe hypoxia. The full range of phenotypic malleability of muscle tissue is demonstrated in people living permanently at high altitude and the hallmark of muscle adaptation to hypoxia is a decrease in muscle oxidative capacity concomitant with a decrease in aerobic work capacity. At the molecular level, training in hypoxia results in an up regulation of the regulatory subunit of hypoxiainducible factor-1 (HIF-1). Possibly as a consequence of this upregulation of HIF-1, the levels mRNAs for myoglobin, for vascular endothelial growth factor and for glycolytic enzymes, such as phosphofructokinase, together with mitochondrial and capillary densities, increased in a hypoxia-dependent manner [19]. Mizuno et al. investigating skeletal muscle adaptations to high altitude and a possible role of physical activity levels showed that mean fiber area was reduced in response to altitude exposure regardless of physical activity which in turn meant that with an unaltered capillary to fiber ratio there was an elevation in capillaries per unit of muscle area. Muscle enzyme activity was unaffected with altitude exposure in active and less active subjects, whereas muscle buffer capacity was increased [27].

\section{3- Skeletal muscle adaptations to microgravity}

Spaceflight results in a number of adaptations to skeletal muscle, including atrophy and early muscle fatigue. Muscle atrophy is observed in a wide range of muscles, with the most extensive loss occurring in the legs, because i) astronauts predominantly utilize their arms to effortlessly push/pull their way about the spacecraft; and ii) they are no longer needed to support the body's weight. Some studies show that a decrease up to $20 \%$ of muscle mass appeared after a relatively brief space missions (one week) [29]. For this reason the understanding of the qualitative and quantitative neuronal mechanisms which control changes in muscle function is crucial for the development of countermeasures able to contrast the loss of skeletal muscle function observed also under simulated microgravity exposure [12]. Numerous studies have examined quantitative and qualitative changes in skeletal muscle, from the whole muscle to the single myofiber from individuals undergoing real and simulated space flight for a few weeks to several months. The losses in muscle mass and function occur rapidly and can be enlarge in magnitude. Some authors report that short-duration ( $<15$ days) spaceflights produce significant losses in muscle mass and strength (5-20\%) [37]. Longer periods, more than six months in spaceflight, losses as high as $40 \%$ in muscle strength has been observed [23]. The decrease in postural muscle fiber size, diminishing of their contractile properties, slow-to-fast shift in myosin heavy chain expression pattern are known to be the main consequences of gravitational unloading. In addition analysis of microarray data revealed that more than two hundred mRNAs were significantly altered by spaceflight, the majority of which displayed similar responses to hind limb suspension, whereas reloading tended to counteract these responses. It is important to note that several mRNAs altered by spaceflight were

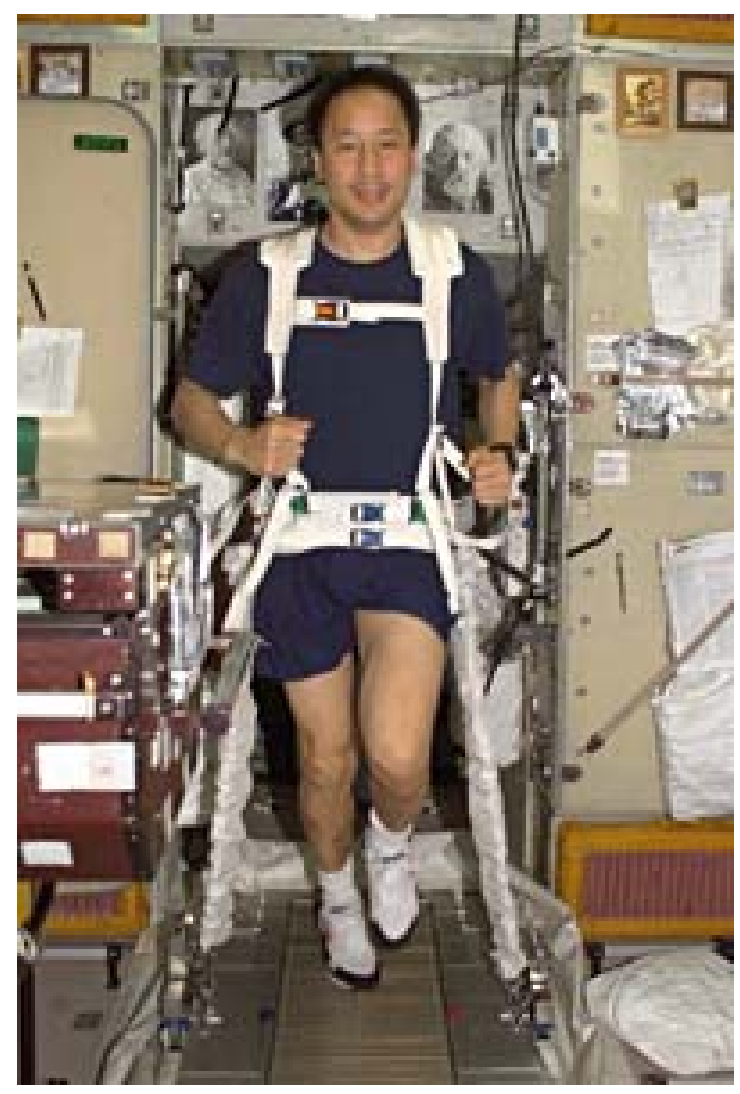

Fig. 5 A regimen of daily exercise in space is crucial to limit muscle and bone atrophy. Astronaut Edward $\mathrm{Lu}$ exercising on a modified treadmill on the International Space Station. The harness and elastic cords provide resistance meant to simulate gravity (NASA: The office of Biological and Physical Research: http://microgravity.hq.nasa.gov/general info / issphysiology_lite.html ). 
Muscle adaptations in extreme environments

European Journal Translational Myology - Myology Reviews 1 (3): 83-90, 2010.

associated with muscle growth [1]. In contrast, there is no change in the cell size or oxidative enzyme activity of spinal motoneurons innervating muscle fibers. Growth-related increases in the cell size of muscle fibers and their spinal motoneurons are inhibited by hind limb suspension [20]. It has been reported that the electromyogram in soleus muscle and the afferent neurogram recorded at level of the spinal cord were decreased in response to exposure to microgravity created during a parabolic flight, as well as similar phenomena were also induced by acute hind limb suspension at $1-\mathrm{G}$ [22].

The Ca2+ role in skeletal muscle atrophic processes due to hypogravity was extensively studied during this last decade. The more important result found was that the resting $\mathrm{Ca} 2+$ level increase in skeletal muscle fibers of hind limb unloaded mice. More recent results showed that the presence of L-type $\mathrm{Ca} 2+$ channels' blockers prevent the $\mathrm{Ca} 2+$ accumulation in the fibers. Thus far, calcium-dependent signaling pathways (like calcineurin/NFAT) may play an important role in the development of some key events observed under artificial or spaceflight unloading [38].

Exercise countermeasures for long duration space crews incorporate aerobic and resistance exercise (Fig. 5). Exercise has been the primary strategy used by the space agencies in an effort to protect cardiovascular, bone, and skeletal muscle status during hypogravity exposure for extended days. The first multifaceted exercise program implemented in space was during Skylab missions in the 1970s. Using a variety of rudimentary exercise equipment, Skylab crewmembers generally maintained cardiovascular capacity while showing decrements in lower leg muscle circumference and performance [34]. Microgravity simulation studies show that resistance exercise alone can be completely efficient to prevent muscle loss, yet crews return from missions with reduced muscle mass and function. Resistance training is a form of strength training in which each effort is performed against a specific opposing force generated by resistance. Some Earth-based studies show that human skeletal muscle size and strength increases when aerobic and resistance training are performed concurrently [39]. For this reason unusual typology of physical training as $20-\mathrm{Hz}$ whole body vibration training was tested to counteract the muscle mass loss induced by hypogravity. Unfortunately daily vibration stimulations, carried out on six volunteers under condition of bed rest, failed to counteract the decrease in leg muscle volume [40]. Several data derived from experiments carried out on animals and humans under condition of artificial hypogravity or during spaceflight of different duration, clearly support the idea that exercise prescription changes are necessary to protect skeletal muscle during space mission especially if it is a long-duration mission. In addition to the risk associated with reduced muscle performance, the decrease in muscle mass and the contractile phenotype shift have risk implications for sensory-motor deficits and skeletal muscle functional integrity. Applying these principles to the manned space program would lead to a more effective exercise program for skeletal muscle, reducing the risk imposed to members of crew.

\section{Corresponding Author}

Giorgio Fanò, Dipartimento di Scienze Mediche di Base ed Applicate (BAMS), Istituto Interuniversitario di Miologia (IIM), Università “G. d’Annunzio” ChietiPescara (Italy). E-mail: fano@unich.it

\section{References}

[1] Allen DL, Bandstra ER, Harrison BC, Thorng S, Stodieck LS, Kostenuik PJ, Morony S, Lacey DL, Hammond TG, Leinwand LL, Argraves WS, Bateman TA, Barth JL. Effects of spaceflight on murine skeletal muscle gene expression. J Appl Physiol 2009; 106: 582-595.

[2] Bae KA, An NY, Kwon YW, Kim C, Yoon CS, Park SC, Kim CK. Muscle fibre size and capillarity in Korean diving women. Acta Physiol Scand 2003; 179: 167-172.

[3] Behm D, Power K, White M, LeDez K, Decker D, Drinkwater E. Effects of hyperbaric (6 ATA) pressure on voluntary and evoked skeletal muscle contractile properties. Undersea Hyperb Med 2003; 30: 103-115.

[4] Bosco G, Zanon V, Camporesi EM. Resistive respiratory muscle training. Undersea Hyperb Med 2007; 34: 145-146.

[5] Bosco G, Yang Zj, Nandi J, Wang Jp, Chen C, Camporesi EM. Effects of hyperbaric oxygen on glucose, lactate, glycerol and antioxidant enzymes in the skeletal muscle of rats during ischemia and reperfusion. Clin Exp Pharmacol Physiol 2007; 34: 70-76.

[6] Camporesi EM, Bosco G. Ventilation, gas exchange and exercise under pressure, in Bennett PB, Elliott DH (eds): The Physiology and Medicine of Diving, 5th edn. London, UK, W. B. Saunders Company Ltd, Best Publishing, 2003, pp 77-114.

[7] Dejours P, Dejours S. The effects of barometric pressure according to Paul Bert: the question today. Int J Sports Med 1992; 13 Suppl 1: S1-5.

[8] Dimai HP. [The effects of chronic hypobaric conditions: skeletal, muscular, and other phenotypic characteristics in high altitude dwellers] Wien Med Wochenschr 2005; 155: 171175.

[9] Fenn WO. The physiological effects of hydrostatic pressure, in Bennett PB, Elliott DH (eds): The physiology and medicine of diving and 


\section{Muscle adaptations in extreme environments}

European Journal Translational Myology - Myology Reviews 1 (3): 83-90, 2010.

compressed air work. London, UK, Baillere Tindall, 1969, p 36.

[10] Ferretti G. Limiting factors to oxygen transport on Mount Everest 30 years after: a critique of Paolo Cerretelli's contribution to the study of altitude physiology. Eur J Appl Physiol 2003; 90: 344-350.

[11] Fisher B, KK Jain, Braun E, Lehrl S. Handbook of hyperbaric Oxygen Therapy. BerlinHeidelberg, ermany, Springer-Verlag, 1988.

[12] Fitts RH, Riley DR, Widrick JJ. Physiology of a microgravity environment invited review: microgravity and skeletal muscle. J Appl Physiol 2000; 89: 823-839.

[13] Flueck M. Plasticity of the muscle proteome to exercise at altitude. High Alt Med Biol. 2009 Summer;10(2):183-93. Review.

[14] Goodall MC, Brown DES. Reneval of relaxing mechanism in muscle fiber systems by hydrostatic pressure. Nature 1956; 178: 14701471.

[15] Hogan PM, Besch SR. Vertebrate skeletal and cardiac muscle, in Macdonald AG (ed): Advances in comparative and environmental physiology, effects of high pressure on biological systems. Berlin-Heidelberg, Germany, Springer-Verlag, 1993 pp 125-146.

[16] Howald H, Hoppeler H. Performing at extreme altitude: muscle cellular and subcellular adaptations. Eur J Appl Physiol 2003; 90: 360364.

[17] Howald H, Pette D, Simoneau JA, Uber A, Hoppeler H, Cerretelli P. Effects of chronic hypoxia on muscle enzyme activities. Int J Sports Med 1990; 11: S10-S14.

[18] Hoppeler H, Kleinert E, Schlegel C, Claassen H, Howald H, Kayar SR, Cerretelli P. Morphological adaptations of human skeletal muscle to chronic hypoxia. Int J Sports Med 1990; 11: S3-S9.

[19] Hoppeler H, Vogt M. Muscle tissue adaptations to hypoxia. Exp Biol 2001; 204(Pt 18): 31333139.

[20] Ishihara A, Kawano F, Wang XD, Ohira Y. Responses of neuromuscular systems under gravity or microgravity environment. Biol Sci Space 2004; 18: 128-129.

[21] Jackson MJ. Redox regulation of adaptive responses in skeletal muscle to contractile activity. Free Radic Biol Med 2009; 47: 12671275.

[22] Kawano F. The mechanisms underlying neuromuscular changes in microgravity environment. Biol Sci Space 2004; 18: 104-105.

[23] Lambertz D, Perot C, Kaspranski R, Goubel F. Effects of long-term spaceflight on mechanical properties of muscles in humans. J Appl Physiol 2001; 90: 179-188.

[24] Margaria R, Camporesi E, Aghemo P, Sassi G. The effect of $\mathrm{O} 2$ breathing on maximal aerobic power. Pflugers Arch. 1972;336(3):225-35

[25] Mathieu-Costello O. Muscle adaptation to altitude: tissue capillarity and capacity for aerobic metabolism. High Alt Med Biol 2001; 2: 413425.

[26] McKenzie JR, Dundas JD. Effect of hyperbaric pressure in isometric muscle contraction and work in intact animals. In Yefuny SN (ed) Proceedings of the 7th international congress on hyperbaric medicine, USRR Academy of Sciences, Moscow, p 383-384, 1981.

[27] Mizuno M, Savard GK, Areskog NH, Lundby C, Saltin B. Skeletal muscle adaptations to prolonged exposure to extreme altitude: a role of physical activity? High Alt Med Biol 2008; 9: 311-317.

[28] Moon RE, Piantadosi CA, Camporesi EM (eds.). Dr. Peter Bennett Symposium Proceedings: Durham, N.C.: Divers Alert Network, 2007.

[29] NASA: Space Life Sciences Research Highligh http://research.hq.nasa.gov/Spaceline_WEB/Spac eLine/nasa/ highlights.html

[30] Park JB, Kim HJ, Kim JC, Lutan R, Kim CK. Muscle characteristics in career breath-hold divers: effect of water temperature. Aviat Space Environ Med 2005; 76: 1123-1127.

[31] Pendergast DR, Lundgren CE. The underwater environment: cardiopulmonary, thermal, and energetic demands. J Appl Physiol 2009; 106: 276-283.

[32] Ranatunga KW, Geeves MA. Changes produced by increased hydrostatic pressure in isometric contraction of rat fast muscle. J Physiol 1991; 441: 413-431.

[33] Ray AD, Pendergast DR, Lundgren CE. Respiratory muscle training improves swimming endurance at depth. Undersea Hyperb Med 2008; 35: 185-196.

[34] Rummel JA, Sawin CF, Michel EL, Buderer MC, Thornton WT. Exercise and long duration spaceflight through 84 days. J Am Med Womens Assoc 1975; 30: 173-187.

[35] Salzano JV, Camporesi EM, Stolp BW, Moon RE. Physiological responses to exercise at 47 and 66 ATA. J Appl Physiol 1984; 57: 1055-1068.

[36] Saunders PU, Pyne DB, Gore CJ. Endurance training at altitude. High Alt Med Biol 2009; 10: 135-148.

[37] Schuenke MD, Reed DW, Kraemer WJ, Staron RS, Volek JS, Hymer WC, Gordon S, Perry Koziris L. Effects of 14 days of microgravity on 
Muscle adaptations in extreme environments

European Journal Translational Myology - Myology Reviews 1 (3): 83-90, 2010.

fast hindlimb and diaphragm muscles of the rat. Eur J Appl Physiol 2009; 106: 885-892.

[38] Shenkman BS, Nemirovskaya TL. Calciumdependent signaling mechanisms and soleus fiber remodeling under gravitational unloading. J Muscle Res Cell Motil 2008; 29: 221-230.

[39] Yang Y, Baker M, Graf S, Larson J, Caiozzo VJ. Hypergravity resistance exercise: the use of artificial gravity as potential countermeasure to microgravity. J Appl Physiol 2007; 103: 18791887.

[40] Zange J, Mester J, Heer M, Kluge G, Liphardt AM. 20-Hz whole body vibration training fails to counteract the decrease in leg muscle volume caused by 14 days of 6 degrees head down tilt bed rest. Eur J Appl Physiol 2009; 105: 271-277. 